\title{
Claudel architecte
}

\author{
Raymond Delambre \\ Directeur de Bibliothèque municipale classée
}

Paul Claudel a exprimé son appétence pour certaines considérations spatiales, tant au sens propre qu'au figuré et le caractère symbolique de son ouvre est connu. Or, espace et symbolisation convergent chez notre auteur.

La question du symbolisme pose, à l'instar du « moderne postmoderne ", celle d'une réflexion épistémologique sur le transfert analogique d'une notion dans un autre champ pour traiter d'un espace distinct... Cette problématique rejoint celle du metatexte, sa possibilité même. L'écrivain contourne ou tranche : sa poésie est metatexte en ce qu'elle constitue un "art poétique», son écriture est instrument de connaissance, toute connaissance étant liée à une croyance'.

En fait, c'est déraison que de chercher à rendre connaissance et croyance incompatibles. Ici réside probablement le motif de l'irritation que suscite volontiers le diplomate. Le monde n'est qu'un « décor » ou une métaphore, partant, l'invisible et le discours sur celui-ci deviennent metatexte...

Claudel n'hésite pas à interpeller «mon ami l'architecte $»^{2}$. Précisément, nous proposons d'étudier les interactions entre la plume claudélienne et l'espace architectural ou architecturé. Focalisons sur l'architecture, les figures de l'architecture, de l'architecte, telles que nous les décelons. Au demeurant, l'architecture comporte un sens excédant, dépassant les limites d'une simple explication matérielle ou matérialiste. En dernière analyse, le criterium architectonique étaye les recherches eschatologiques. Les signes claudéliens nous inviteraient à une sémiotique de l'architecture.

Légion $^{3}$ sont les allusions architecturales : à nous de préciser, analyser, féconder les évocations. De fait, les "sites claudéliens".- textuels - reçoivent et stimulent un questionnement sur la production de l'espace littéraire et la construction des perceptions architecturales. Nous dévoilerons les tenants et aboutissants, voire les arcs-boutants étayant le « traité d'architecture claudélien ». 
Le dramaturge s'interroge sur les " modalités de l'habité ", ou plutôt de l'habitant. Objet et sujet, habitation, habité, habitant sont intimes : le poète tente de transcender l'habitation, mondaine, corporelle, afin de révéler le sujet, l'occupant, temporaire... L'hérö̈ne accomplie, Prouhèze, dans ses efforts pour convaincre Rodrigue de la laisser quitter son enveloppe charnelle, se transformer en étoile, est emblématique. Finalement, tout est décor, ou « compartiment ». La question du rapport au monde, de la capacité ou non, mieux, de l'opportunité de l'habiter est directement abordée. Nous forgeons la formule « déficit d'habitabilité »...

Sachant que les valences de l'habitation, de l'habitant sont réversibles... "Ce n'est pas l'esprit qui est dans le corps, c'est l'esprit qui contient le corps, et qui l'enveloppe tout entier." »

\section{L'architecture, fundamentum fundamenti; l'œuvre claudélienne : un monumentum, sinon ambigu, en tout cas ambivalent}

Claudel a eu plusieurs fois l'occasion de louer la vertu cardinale de l'architecture. "Souviens-toi, homme, que tu es pierre, et que sur cette pierre j'ai établi le fondement d'un édifice indestructibles! »

Conjointement, nous savons l'importance que l'ambassadeur attache au texte. Celui-ci est à ériger en fondement... Or, nous verrons que le poète associe bâtiment et texte. Certains écrits sont proprement monumentaux...

Le Soulier de satin apparaît certes comme un monument de " construction littéraire ". Paradoxalement, ou logiquement, ce « jumbo théâtral » est confronté aux difficultés de la représentation spatiale, sur la scène, et temporelle. $\mathrm{La}$ " représentabilité » du théâtre, singulièrement du Soulier, sa " mise en espace ", sinon sa mise en scène sont interpellés. Néanmoins, on trouve des didascalies.

\section{Leçons chinoises sur l'espace, sur l'architecture...}

Le Soulier, œuvre monumentale, s'amenuisant en un Rodrigue démembré, qui ne vaut plus un sou, pratique la dialectique du microcosme et du macrocosme, illustre celle-ci. Réminiscence d’un procédé chinois : dès 


\section{Claudel architecte}

l'antiquité, la Chine concevait la dialectique du macrocosme et du microcosme. Xiao Zhong Jian Da, Da Zhong Jian Xiao : « au sein du petit se voit le grand, au sein du grand se voit le petit $»^{6}$.

De fait, notre auteur bénéficie de leçons chinoises. La symbolique du Céleste Empire est édifiante. Ainsi : Bu Lao Song, «le pin toujours jeune $»^{7}$. Cette symbolisation n'a pu laisser indifférent le poète...

Le grand voyageur s'intéresse au jardin asiatique, architecturé, notamment par la taille. Le jardin est élevé au rang de microcosme en Chine. Sa petitesse relative est d'autant plus révélatrice que l'Empire du Milieu ne manque pas d'espace : partant, le jardin microcosmique relève d'une intention délibérée, voire didactique, spirituelle. Ce ne sont pas des conditions « objectives » spatiales qui dictent la configuration du jardin ${ }^{8}$.

\section{Claudel en Asie, l'Asie en Claudel...}

En particulier, une surprenante manifestation du paysage émane de la Chine. Au demeurant, Claudel livre une pénétrante observation, s'agissant $\mathrm{du}$ toit. Le poète traite de l'architecture en invoquant une ultima ratio typiquement chinoise, anticonformiste, légèreté et apparente lourdeur étant associées.

Si chez l'exégète de la Bible l'environnement vaut pour ce qu'il signifie, notre écrivain prend davantage au sérieux que Victor Segalen ce qui l'entoure, ne le ravale pas en un prétexte ou alibi, parce que le " décor" fait signe à celui qui sait l'écouter... Le sinologue doit probablement être aussi sismologue. Mutatis mutandis, le créateur, pour se croire pleinement chrétien d'Occident, évoque tout de même son antipode...

A la différence de la pensée occidentale, l'intelligence chinoise est centrée sur l'espace. Le temps en est simplement un représentant. Harmonisation du temps et de l'espace... Or, la dialectique du temps et de l'espace agit sur la plume claudélienne. Souvenons-nous du « quand sera$c e$ ? " émis par Rodrigue éploré aux pieds ${ }^{9}$ de Prouhèze annonçant son départ céleste au paradis des chiffres et astres...

L'Empire du Milieu est le pays du "paysage-idée ${ }^{10}$ » : l'espace n'y est pas seulement réduit au visible. Cette conception a pu contribuer à la défense de l'" invisible ${ }^{11}$ " chez le "poëte », qui a su se déprendre de la «fureur oculaire ${ }^{12}$ ». 


\section{Raymond Delambre}

\section{Le « vide » et le plein}

L'Oiseau noir dans le soleil levant se réfère à la dialectique du plein et du vide. "Le paysage est constitué de deux triangles, l'un massif et nourri, l'autre évidé et allongé jusqu'à l'exténuation [...] Dans tout tableau, il y a un équilibre du plein et du vide, le yang et le yin, le mâle et la femelle ${ }^{13}$."

De même, dans une conférence tenue en 1925 à Madrid, Lyon, Bruxelles... "Les Japonais apportent dans la poésie comme dans l'art une idée très différente de la nôtre. La nôtre est de tout dire, de tout exprimer [...] Au Japon au contraire sur la page, écrite ou dessinée, la part la plus importante est toujours laissée vide. "

Notre auteur visa directement le Dao De Jing. L'enseignement de Lao Zi lui-même fait référence à l'architecture. Mieux, le Maître du Dao applique précisément sa théorie du vide au bâtiment : "On ménage des portes et des fenêtres pour une pièce

Ce vide dans la pièce en permet l'usage ${ }^{14}$.

Dans La Cathédrale de Strasbourg, le pèlerin analyse le vide. "Les grands vides dont le rapport entre eux constitue la matrice religieuse et le vaisseau de notre comprésence en Dieu ils n'ont pour rôle que de modeler autour de nous l'invisible ${ }^{15}$. L'architecte d'aujourd'hui parle des «circulations», "flux », de la « H.S.P. $»^{16}$.

Le poète décline notre dialectique du vide et du plein en matière d'art poétique, dans la Philosophie du livre. « Le blanc n'est pas [...] seulement pour le poème une nécessité matérielle imposée du dehors. Il est la condition même [...] de sa vie et de sa respiration [...] C'est cette importance de la page, c'est cette idée du rapport nécessaire entre le contenu poétique et son contenant matériel, entre ce plein et ce vide, qui avait inspiré à Stéphane Mallarmé lidée de sa dernière cuvre, de ce grand poème typographique [...] Un coup de dés jamais n'abolira le hasard [...] Et je me souviens tout en haut d'une grande page blanche, de ce seul mot plume en caractères italiques, comme un flocon de neige ou comme l'unique duvet qui reste d'une colombe disparue ${ }^{17}$."

Hélas, l'époque contemporaine est celle de la surcharge, affectant tant les corps que les villes et les feuilles : surcharges pondérale, monumentale, graphique... Au demeurant, le déficit d'espace vide entraîne la claustrophobie... 


\section{Claustrophobie... et probablement agoraphobie}

Le déficit d'habitabilité caractérisant trop souvent l'espace humain est magnifiquement illustré par la vision du "compartiment ", oppressant l'homme, manifestée par Rodrigue. Le champ sémantique du " compartiment » est bienvenu : conjointement à l'espace clos, resserré, voire à partager avec d'autres, il évoque le compartiment de train, le wagon, le chemin de fer. Ou évoquait : le contemporain est volontiers amnésique... Or, les wagons vont dans la même direction : le chauffeur ${ }^{18}$ n'est pas dans chacun des wagons. Le compartiment est certes un « appartement sur roues ", mais à quoi bon en changer, sachant que les wagons sont tirés ${ }^{19}$ par une force qui les dépasse?

Le compartiment s'emboîte dans l'architecture d'Alberto Giacometti... « Figurine dans une boîte entre deux boîtes qui sont des maisons $»^{20}$. Les " maisons littéraires » de Claudel, même si elles semblent s'élargir aux dimensions du monde, dans le Soulier, n'échappent à la claustrophobie que grâce à l'adjonction d'une dimension surnaturelle à l'espace platement physique.

La sagesse finale de Rodrigue, satisfait de son abaissement social et de son amputation corporelle, dont le « compartiment » est de plus en plus réduit, entre remarquablement en correspondance avec la leçon de Su Dongpo : "à l'île de Hainan, je me suis vu entouré [...] par l'eau [...] Quand pourrai-je sortir de cette île?21 [...] l'univers est au milieu de l'eau, les pays au-delà de la Chine sont au milieu d'océans, la Chine est au milieu de mers moins importantes. Nous vivons tous sur une ille ${ }^{22}$. "Nous vivons tous dans un compartiment, dirait Rodrigue au dénouement - comme on délie, délace une chaussure - dans le Soulier... Soulier doublement dénoué : Prouhèze empêchée de pécher, mieux, libérée de cette tentation, Rodrigue quant à lui délivré de ses nombreux attachements, de ses attributs socioéconomiques.

Le « catholique errant » - nous baptisons ainsi le diplomate enseigne dans la Maison d'Antonin Raymond à Tokyô que les demeures occidentales ne sont que "nos casernes locatives $»^{23}$. Raymond et sa maison constituent un antidote au casernement.

Le déficit d'habitabilité rejoint l'éloge de la fuite, Christophe Colomb fuyant. "Je voudrais être tellement parti que le retour soit impossible»...

La mobilité, palliatif destiné à rendre supportable l'inhabitable condition humaine, se traduit précisément sur le plan architectural. Le 


\section{Raymond Delambre}

grand voyageur loue, à l'instar de François Cheng, l'envolée du toit : «les architectes chinois, amis des courbes, ont toujours eu souci de donner des ailes à leurs toits [...] le lieu de l'Homme se situe entre Terre et Ciel [...] aux yeux du sage, le but de l'existence est moins de s'établir que de s'évader ${ }^{24}$."

L'architecture peut doter l'homme d'ailes. Elle l'explique, aussi.

\section{L'architecture, heuristique de l'homme}

A travers Jean Charlot, le critique d'art applique à l'organisme la métaphore architecturale : "le corps humain isolé est un édifice qui s'appuie solidement sur ses fondations, qu'il s'agisse $d u V$ de l'aine ou de la double masse du trochanter $»^{25}$. Ambivalence humaine, sinon ambiguïté... «La vérité est que l'être humain est bâti [...] à deux étages; au premier, ce que les Allemands appellent le "bel-étage ", il y a un Bourgeois naïf [...] dans le sous-sol, il y a un original $»^{26}$.

Il n'est pas indifférent de constater que notre poète, s'il raisonne en termes de superstructure et d'infrastructure, même de soubassement, s'il ne s'intéresse pas qu'au piano nobile de l'homme, ne verse pas dans la « psychologisation ${ }^{27}$. Son analyse est davantage de nature politique. Le catholique errant poursuit, avec un Christ révolutionnaire ${ }^{28}$. "Le Christ, à chaque page de Son livre, répète qu'il est venu apporter "le glaive", "la division", "l'arrachement" ${ }^{29}$. Cette vision, peu ou prou apocalyptique, se voit via l'urbanisme claudélien, la Ville souffrant d'un radical déficit d'urbanité et promise à l'autodafé, qu'on espère rédempteur. L'exégète ressent la dualité christique, Jésus étant à la fois la pierre d'achoppement et la porte.

Traitant d'un autre peintre, Henry de Waroquier ${ }^{30}$ file la métaphore architecturale organique, l'architecture conférant finalement à l'homme sa spiritualité : "si le corps humain est un temple [...] le visage, la tête, en est à la fois le portail et le sanctuaire. Il serait trop long d'en étudier ici les organes sacrés, le front sublime, la double arcade divisée par le pilier nasal, et par derrière ces deux yeux qui voient à la fois et qui regardent $n^{31}$. De Jean Charlot à Henry de Waroquier, notre architecte passe d'une architecture éclaircissant la nature matérielle, voire la solidité du corps, à une architecture spiritualisant celui-ci.

La verticalité caractérise notamment l'architecture et l'homme, ainsi que l'arbre. 


\section{Claudel vertical}

La verticalité est essentielle. Le dramaturge a pu jouer des plans horizontal et vertical, en imaginant des scènes verticales; l'absence de rideau de scène, typique d'un certain théâtre asiatique, susceptible d'abolir la frontière entre acteurs et spectateurs, a pareillement influencé le voyageur. Au demeurant, Arbor Vitae... "L'arbre seul, dans la nature, pour une raison typifique, est vertical, avec l'homme ${ }^{32}$."

S'agissant de Jeanne d'Arc... "Pour comprendre une vie, comme pour comprendre un paysage, il faut choisir le point de vue, et il n'en est pas de meilleur que le sommet. Le sommet de la vie de Jeanne d'Arc, c'est sa mort, le bûcher de Rouen. » De même que c'est la flamme qui fait, i.e. légitime la bougie, de même c'est le haut qui explique le bas : sed Ego qui elegi vos...

Le Soulier a vocation d' «élargir le monde». Ne comporterait-il pas l'enseignement suivant? s'il s'agit pour Rodrigue d'élargir le monde, nonobstant circuler d'un territoire à l'autre, d'un continent à l'autre se résume, in fine, à changer simplement de "compartiment ». L'élargissement serait donc à inscrire dans le champ sémantique des prisonniers : seule l'aspiration vers le haut, la verticalité inspirée par la spiritualité libère.

L'aspiration verticale est déclinée en architecture. Le critique use volontiers des catégories architecturales en traitant de la peinture. La verticalité unit art de la construction et art pictural. La Peinture espagnole évoque «cette plate-forme verticale qui est la toile tendue $e^{33}$.» A propos de Jean Charlot ${ }^{34} . .$. «Il lui faudrait de grands espaces à remplir, pourquoi pas tout ce panneau immense entre l'Atlantique et le Pacifique? Quelle joie de faire passer tout un monde dans le domaine de la verticale et de grimper à une grande échelle $e^{35}$ ! "Chez Henry de Waroquier, la " cascade est faite de lumière solidifiée par une distance virtuelle. Tout conspire à la verticale, l'homme s'est chargé d'achever le vou tellurique [...] la plus vieille attitude de l'humanité. On songe aux pierres levées, aux quilles de l'île de Pâques, aux stèles chinoises [...] aux stoupas bouddhiques ${ }^{36}$. »

Conjointement au «Claudel vertical », nous pourrions forger la formule du «Claudel perpendiculaire », en vue de positionner l'écrivain dans l'espace occupé par les prises de position : de fait, les positions claudéliennes sont perpendiculaires ${ }^{37}$, en "opposition ». Le catholique errant, pourfendeur ${ }^{38}$, après le Christ... Précisément, le poète assène des coups bien sentis à l'encontre des « B.T.P. ${ }^{39} \ldots$ 


\section{Raymond Delambre}

\section{L'architecte et le peintre : architecture de la peinture, peinture de l'architecture...}

Le prisme architectonique constitue la méthode d'analyse picturale, qui donne du volume à la "façade " des tableaux peints par Henry de Waroquier. "Le plan vertical [...] n'est que l'aboutissement d'un travail rétromotif de l'expression. Ce n'est qu'une façade, et derrière la façade, il y a la substance $[. .$.$] le volume { }^{40}$. " Du plan à la façade, du plan au volume...

Que recouvre au fond la notion de "façade »? L'auteur s'intéresse effectivement à celle-ci, dont on sait qu'elle est débattue. «Une façade [i.e.] ce qui au nom de l'édifice prend vue sur de l'étendue, ce qui-le tableau est là devant nous, accroché à son clou - au nom de l'invisible prend vue sur de l'invisible [...] Il s'agit de résister au temps par le moyen d'une nécessité intrinsèque. Et quel recours à cet effet meilleur qu'à la géométrie? C'est ainsi que le moyen se défend à la fois contre l'arbitraire et contre l'oubli par le moyen de la prosodie. L'art de Waroquier est un arrangement ${ }^{41}$ de solides où les éléments choisis se sentent et se ressentent calés l'un par l'autre ${ }^{42}$."

La prosodie ou la géométrie, voire la narration, octroient à l'architecture et à la peinture leur sens, qui «cale» les éléments et les rend solides. Dans une perspective chinoise... "Le paysage n'est plus rencontre avec le hasard, c'est un cas, c'est une situation dramatique [...] et surtout c'est une affirmation ${ }^{43}$."

Est-il légitime de traiter la peinture sur le mode architectural? oui, puisque « Tout est devenu architecture. La couleur ne fait que qualifier le bris de la surface par le complexe intérieur ${ }^{44}$. " Au demeurant, le peintre et l'architecte firent bon ménage.

Le peintre en tant que tel devient, sous la plume claudélienne, un bâtisseur... "De même qu'une maison consiste de briques, d'acier, de bois et de verre dans une certaine proportion et que tout cela fait un solide habitable, de même que d'os et de chair dans une certaine proportion est fait un être humain, de même un tableau est un certain ensemble de lignes, de plans, de volumes et de tons. Nous exprimerons cette idée en disant que Jean Charlot est un constructeur, un compositeur, que [...] la sensation de la masse et du poids autour d'un invisible fil à plomb n'est jamais absente $»^{45}$.

Mieux, la couleur composante architectonique... " La couleur [...] est un élément architectural et le jaune par exemple dans une certaine proportion soutient le rose, comme un arc supporte le bandeau. J'exprimerai toute ma pensée en disant que la peinture, c'est la beauté qui arrête le temps et qui d'une mélodie $e^{46}$ fait pour nous un spectacle ${ }^{47}$. " 


\section{Claudel architecte}

$\mathrm{Au}$ total, les conceptions picturales de l'écrivain sont volontiers empreintes de considérations architecturales. L'architecture tend à expliquer la peinture, vaut en quelque manière méthode, voie d'accès, moyen d'accéder à la connaissance. Conjointement, elle est aussi une fin, une «matière ». Le poète osant se présenter, architecte. "Les ombres de Reims et de Laon [...] n'ont-elles pas touché mon berceau et limité mon horizon de poète, de croyant et d'architecte ${ }^{48}$ ?"

\section{L'architecture claudélienne}

Tout d'abord, distinguons l'architectonique de la sculpture... "L'architecture pétrifie la proportion, et la sculpture l'attitude ${ }^{49}$. "La Cathédrale de Strasbourg permet d'évoquer la "châsse spirituelle, solidification du mystère par l'architecture » ${ }^{50}$.

L'église définit" l'architecte : "l'ingénieur en spiritualité que j'imagine $"{ }^{52}$. Le syntagme maison de prière est révélateur, associant une " réalité concrète ${ }^{53}$, la maison, à un acte spirituel : importance du bâtiment, du réceptacle... De même, s'agissant de l'ingénieur, notre auteur marie le technicien et la spiritualité. L'alliance du spirituel et du matériel est chère à Claudel, déclinée en matière architecturale.

Il n'est pas indifférent de constater que le personnage emblématique de Pierre ${ }^{54}$, Pierre de Craon - les noms propres, les toponymes sont naturellement justiciables d'une onomastique - est l'un des premiers protagonistes claudéliens. Or, Pierre est architecte... Cette figure apparaît à la fois dans la Jeune fille Violaine et l'Annonce faite à Marie.

Pierre, architecte métaphorique, pierre symbolique, expliquée par José-Maria Sert ${ }^{55}$ et sa cathédrale : "le Romulus et le Rémus de la Cité nouvelle soulèvent et soutiennent de leurs mains et de leurs épaules la Pierre fondamentale.

Ainsi le peintre avec l'architecte ${ }^{56}$."

La convergence entre architecture claudélienne et spiritualité doit se mettre en exergue. La septième Affirmation profere : "Souviens-toi, homme, que tu es pierre et sur cette pierre Je bâtirai mon église! [...]

Et les portes de l'enfer ne prévaudront pas contre elle ${ }^{57}$ !"

L'architecte inspiré, spirituel, œuvre à l'instar de l'âme sur le corps, de la flamme sur la bougie : ce ne sont point le physique, la bougie qui sont au principe, mais l'esprit, l'ardeur. Partant, l'apprenti architecte 


\section{Raymond Delambre}

dans la version initiale de l'Annonce aspire à devenir un "ingénieur en spiritualité » - transcendant l'oxymore constitué par ce nouveau titre "Car ${ }^{8}$ l'artiste païen faisait tout $d u$ dehors, et nous faisons tout de par dedans comme les abeilles,

Et comme l'âme fait pour le corps : rien n'est inerte, tout vit $\aleph^{59}$.

La Philosophie, et plus particulièrement la Religion du signe marient spiritualité et Céleste Empire. «Par les proportions de sa cour et des péristyles qui l'encadrent, par les larges entrecolonnements et les lignes horizontales de sa façade, par la répétition de ses deux énormes toits, qui d'un mouvement un relèvent ensemble leur noire et puissante volute [...] l'édifice, appliquant les seules lois essentielles de l'architecture, a l'aspect savant de l'évidence, la beauté, pour tout dire, classique, due à une observation exquise de la règle ${ }^{60}$. " $\mathrm{Au}$ demeurant, la Religion souligne l'alliance de la spiritualité et de l'écriture chinoise ${ }^{61}$, évoquant la « piété des Chinois à l'écriture", même la « religion scripturale " ${ }^{62}$.

En contrepoint, les Conversations dans le Loir-et-Cher objectivent l'idéologie du gratte-ciel ${ }^{63}$, ravalé à un piètre rang : $l^{\prime}$ « $I . G . H »$, expression des professionnels, i.e. l' "immeuble de grande hauteur » ${ }^{64}$, n'est qu'une accumulation de tiroirs... La création du "Loir-et-Cher » est à interpréter en une leçon de défiance à l'encontre de la géographie, de l'espace simplement matériel, ou administratif au sens des divisions départementales ou régionales ${ }^{65}$ : autant lui préférer une géographie imaginaire, la géographie «scientifique » n'étant pas de plano heuristique, ni légitime. La verticalité préconisée ne doit pas se traduire de façon caricaturale et architecturale par l'érection du vulgaire gratte-ciel.

La Sagesse elle-même déplore le néant "moderne ", peuplé de tours... "C'est le désert d'hommes et d'âmes!

Ici apparaissent peu à peu les gratte-ciel6."

La notation s'avère pertinente, s'agissant tant de la cité que de l'âme : contrairement à ce que donnerait à penser un certain "sens commun architectural » - nous baptisons les poncifs composant la doxa architecturale - les gratte-ciel ne favorisent nullement la densité; tout à rebours ce que nous appelons les « villes à tours " provoquent des béances dans le tissu urbain, de vastes espaces vides étant ménagés à la base des bâtiments élevés. Conjointement au fait que nombre de gratte-ciel hébergent des bureaux, dépeuplés dès que les "heures de bureau " ont fui...

L'auteur, volontiers doctrinaire architectonique, se prononce en faveur de l'ornementation. En accord avec le précepte formulé en 1853 par 
John Ruskin... "L'ornementation est l'origine de l'architecture ». Le voyageur se démarque, sur un plan proprement esthétique, du jansénisme et de sa doctrine esthétique. "Tout le plaisir qu'on prend des choses visibles diminue d'autant la vie de la grâce ${ }^{67}$.

Dans cette perspective, on trouve chez notre poète des remarques architecturales comparables aux saillies élaborées par Henry-Russel Hitchcock... «A Kiefhoek [...] on demanda à Oud de réaliser une église [...] l'auditorium principal [...] ressemble absolument à une boîte qui tient sa place parmi les rangées de maisons, n'offrant aucune [...] expression de son but spécifique - on pourrait tout aussi bien le prendre pour un garage ${ }^{68} . "$

Le catholique errant et la Bible ne peuvent ravaler une église en "garage ", puisque " tout ne bâtit pas". "Tout est permis, mais tout ne profite pas. Tout est permis, mais tout ne bâtit pas ${ }^{69}$. "Si l'on nous autorise une formule: on ne bétonne pas impunément...

Enfin, notre auteur évoque la genèse, ou l'une des genèses de l'architecte, via la figure de l'orfèvre... « Voici à l'ouvre à travers les siècles ce sculpteur et cet architecte qu'est l'Orfevre ${ }^{70}$."

\section{Les pierres de Claudel $^{71}$}

La Mystique des pierres précieuses nous fournit un florilège des pierres aimées... "Ainsi cette pierre merveilleuse dont parle Buffon, et que j'aime autant ne pas identifier, et qu'il appelle le giraso ${ }^{2}$."

La pierre est polysémique, pierre d'achoppement ou support ${ }^{73}$, couronnement et fondement : "couronnement de l'édifice, la pierre spirituelle en est en nous la fondation: "Je disposerai tes pierres précieuses par ordre, dit Isaïe, et je te fonderai sur les Saphirs" " ${ }^{74}$.

La pierre se fait encore ouverture ${ }^{75}:$ «dans l'Apocalypse, les portes de Jérusalem sont comparées à des perles et [...] la Vierge elle-même est appelée la Porte orientale ${ }^{76}$. "L'Apocalypse enseigne effectivement que la Jérusalem céleste " descendait telle une pierre très précieuse, comme une pierre de jaspe cristallin. " L'importance biblique attachée aux pierres explique probablement l'appétence de l'exégète pour celles-ci.

Au demeurant, la pierre de touche vaut criterium de la valeur, aux sens propre et figuré. Passons de la pierre au verre... 


\section{La maison de verre claudélienne}

Sur les Vitraux des cathédrales de France, Claudel se prononce. "Il n'y aucune raison de ne pas nous servir du verre pour réaliser dans le courage et dans la joie des poèmes lumineux et instructifs [...] pourquoi n'imagineraiton pas une église où les parois de verre suivant des passages calculés de dessins et de couleurs seraient consacrés au développement d'une seule idée ou thème? J'avais imaginé par exemple celui du Fleuve qui tient une telle place dans les Livres saints ${ }^{77}$. " L'écrivain ${ }^{78}$ promeut même la Magie du verre ${ }^{79}$...

Afin de magnifier le verre, l'oxymore de la «fongibilité durable» est invoqué, ainsi que la référence au noble élément aquatique ${ }^{80}$. " J'ai toujours tellement aimé le verre, qui est une espèce d'eau solidifiée $e^{81}$, tangible, intangible entre nos doigts, une fongibilité durable, une contenance spirituelle ${ }^{82}$ ! La «contenance spirituelle », oxymorique et poétique, fait écho au Soulier ${ }^{83} . .$.

Finalement, le verre ou plutôt sa magie éclairent le regret de l'écrivain, son désir d'architecte... « Le poète habitué à travailler sur une matière imaginaire comme il aimerait sur ses vieux jours à se faire verrier, architecte de l'un de ses châteaux toujours suspendus suivant l'inclinaison du rayon extérieur entre l'existence et le rêve! Qu'on lui fasse une commande pour l'exposition qui se prépare et son projet est prêt ${ }^{84}$."

$\mathrm{Du}$ verre passons à une thématique en soi plus profonde : celle de la lumière ${ }^{85}$.

\section{Leçons de Lumière ${ }^{86} .$.}

Plutôt que de nous assombrir, voire nous assourdir par des Leçons de ténèbres, Claudel souhaite dans L'église que l'architecte ait à l'esprit la consigne : lux lucet in tenebris... "Par le moyen de la nuit il faut vous donner à habiter de la lumière ${ }^{87}$. "L'instruction est formelle, consacrant le caractère divin de la lumière... "Faire de la lumière, pauvres gens, c'est plus difficile que de faire de l'or ${ }^{88}$."

La Maison d'Antonin Raymond révèle au diplomate, sur un plan architectonique, l'importance de la "lumière naturelle ", expression des spécialistes. "Rien de plus triste que notre fenêtre rectangulaire qui a l'air moins faite pour respirer l'air et la lumière que pour les repousser de son multiple bouclier de verre avare et de rideaux ${ }^{89}$. " 
L'exégète ne pouvait qu'être sensible à la lumière en architecture, cruciale, les photons étant en quelque sorte supports de la foi : "un document, constituant un code [...] c'est la justification que donne de la cathédrale l'évêque Suger dans son Liber de administratione sua gestis [...] la lumière qui pénètre à flots dans les nefs sombres (la structure des murs permettant d'offrir à la lumière une voie d'accès si large) doit représenter l'effusion de l'énergie créatrice divine, et cela sur la base des textes néoplatoniciens et sur une équivalence codifiée entre lumière et participation de l'essence divine ${ }^{90}$.

Plus techniquement, notre "ingénieur en spiritualité " analyse la diffraction. "Dans la cathédrale ancienne la couleur, épousant la lumière, va $d u$ dehors vers le dedans, ou plutôt elle est la lumière même, suivant que le verre en la qualifiant lui permet de passer, disséminée et répartie $e^{91}$.

L'église définit même l'architecte comme devant illuminer à partir $\mathrm{du}$ « noir absolu» : "pareil au graveur qui part du noir absolu, l'architecte que j'essaye ici d'encourager à l'existence ${ }^{92}$ aura pour tâche de qualifier le contenu de ce vase saint dont il a entrepris la paroi et de construire, non plus seulement sur de l'acier et des briques, mais sur l'ombre même en tant que matériau essentiel et le sentiment nuancé et développé par elle, son système de solides [...] cette lumière jadis nous n'étions capables que de la capter, de l'utiliser telle quelle, mais [...] nous sommes maîtres de la produire, de la faire jaillir ou émaner de la substance même de l'édifice, de la manier comme l'instrument le plus subtil, de la diriger comme une attention spirituelle appliquée dans une juste perspective de plan divers à un objectif essentiel. Le théâtre, le cinéma ${ }^{93}$, l'éclairage urbain, nous fournissent $[. .$.$] des leçons dont$ il est surprenant que l'église n'ait pas encore profité. Les anciens maçons n'étaient pas si timides qui essayaient naïvement pour modeler la Madone aux yeux de ses enfants de faire tomber sur elle la clarté d'un oculus ${ }^{94}$." L'éclairement devient ainsi spirituel. En rapport avec l'architecture sacrée.

\section{Variations sur le temple claudélien...}

Claudel accorde une importance cruciale au temple. Au demeurant, le point d'orgue que le discours architectural claudélien confère à l'église s'inscrit dans une tradition, religieuse, littéraire et urbanistique.

Il ambitionne même de tirer les plans de l'église idéale. L'église s'analyse en un "cahier des charges ", voire en une " étude de 
programmation fonctionnelle", si l'on veut employer les expressions techniques adoptées par le maître d'ouvrage. "Essayons [...] de déterminer ici $[. .$.$] quelques-unes des conditions physiques de la prière et les moyens$ matériels qui seraient le plus propres à leur forme donner. De ces conditions la première et la plus essentielle est le silence, je dis silence de la vue, de l'ouïe et de tous les sens ${ }^{95}$ [...] Il faut aussi faire sauter la courroie de la poulie [...] interrompre ce mouvement automatique qui intarissablement apporte les images à l'imagination [...] C'est bien assez du bruit intérieur [...] Il faut proscrire les chaises et les remplacer par ces pews solides et confortables qui existent dans les églises américaines ${ }^{96}$. "

La leçon ci-dessus, découverte dans un humble Appendice, d'application rapide : "Voilà ce que j'appelle les dispositions prochaines" ${ }^{97}$." L'église traite du long terme architectural, de façon " concrète ", le progrès s'inspirant des lointains asiatiques. "Occupons-nous maintenant des dispositions éloignées qui concernent l'édifice et son architecture. Quand j'habitais la Chine et le Japon j'ai souvent admiré le plan des temples qui ne permet pas au fidele d'arriver directement à l'idole ou à la tablette, mais le fait circuler par toutes sortes de biais et de détours, ponts, portes, galeries, escaliers, ouvertures coupées par des écrans, haltes sur des paliers secondaires, de manière à donner à l'âme le temps de se composer grâce aux préparations qui sont insinuées ${ }^{98}$ au corps ${ }^{99}$."

L'art du détour ne rapprocherait-il pas l'architecture de la littérature? Sinon la fiction, en tout cas la narration, le récit ne seraient-ils pas des chemins détournés? L'Odyssée, prototype du récit " occidental », ne vaut-elle pas, essentiellement, par les détours qu'inflige Homère à Ulysse? L'architecte, à l'instar de l'artiste, gagnerait probablement à (re)lire Benjamin Constant, se défiant de l'apparente pertinence — nous dirions limpidité superficielle - de la ligne droite...

Le détour cultivé par l'architecture chinoise explique l'appétence pour l'Asie. Elogieuse, la Connaissance de l'Est, dédiée au Céleste Empire, encense le temple... "Le lieu religieux ici n'enferme pas, comme en Europe, unique et clos, le mystère d'une foi et d'un dogme circonscrits [...] il établit un certain milieu, et, suspendu en quelque sorte au ciel, l'édifice mêle toute la nature à l'offrande qu'il constitue ${ }^{100}$. » L'emprunt au paysage est ainsi évoqué. De fait, la construction en Empire du Milieu interagit avec son environnement; e.g. une fenêtre cadre (diverses acceptions) le panorama. En rapport avec la plume claudélienne, poétique du monde, célébrant une vaste adhésion à l'univers... 


\section{Claudel architecte}

Sur les Vitraux, formule de la «Bible latente ${ }^{101}$. E.g. : la « Bible de pierre ", justiciable d'une lecture.

De l'architecture en " trois dimensions », l'exégète passe à la Philosophie du livre, à la Religion du signe, i.e. à la fois à un monde de surface, plane - celle du livre - et à une autre dimension, au delà des trois dimensions... Le « second poème est la description d'un de ces temples confucéens qui [...] ne comportent aucune idole, mais seulement une exposition solennelle de ces caractères qui servent de support perpétuel à l'âme des Sages défunts ${ }^{102}$. Réminiscence chinoise. Mariant caractères et temples, littérature et architecture...

L'Oiseau noir cultive la métaphore de la page, afin de commenter l'architecture asiatique : «ce n'est jamais l'édifice, aussi doré qu'il puisse être, qui me paraît, comme en Europe, l'essentiel. Ce n'est jamais qu'une cassette, un encensoir déposé dans un coin pour faire sentir l'immense solennité de la nature et, si je peux dire, "la mettre en page " ${ }^{103}$. " Le voyageur a bien compris la spécificité valorisant le milieu, empruntant au site : Feng Shui ${ }^{104} \ldots$

\section{La spatialité du texte}

Notre architecte relie directement page et architecture, dans la Philosophie: «ce que j'appelle la page, une architecture de lignes contenue et déterminée par un cadre ${ }^{105}$. » Claudel pousse plus loin encore l'assimilation, avec les «édifices typographiques », expression octroyant du volume et de la verticalité — dont on a vu qu'elle est chère à l'écrivain à la planéité et à l'horizontalité attachées au support typographique. «Ces édifices typographiques sont aussi beaux que les façades de Palladio et de Borromini ${ }^{106}$."

L'écrivain explique l'architecture textuelle via notre dialectique du plein et du vide. "Cette écriture [...] en horizons superposés, en masses carrées qui viennent successivement se présenter au regard dans le portique du papier, en pages. La page consiste essentiellement en un certain rapport du bloc imprimé ou justification et du blanc, ou marge. Ce rapport n'est pas purement matériel, il est l'image de ce que tout mouvement de la pensée [...] laisse autour de lui d'inexprimé, mais non pas d'inerte, mais non pas d'incorporel, le silence environnant d'où cette voix est issue et qu'elle imprègne à son tour, quelque chose comme son champ magnétique. Ce 


\section{Raymond Delambre}

rapport entre la parole et le silence, entre l'écriture et le blanc, est la ressource particulière de la poésie, et c'est pourquoi la page est son domaine propre » ${ }^{107}$.

Nous ne pouvons citer davantage, sauf à "finir " par une antiphrase, à l'instar de l'ambassadeur, abordant l'illustration. " J'ai fini ${ }^{108}$, et ce n'est pas sans regret ${ }^{109}$ que je suis obligé de laisser de côté certains aspects [...] l'illustration par exemple [...] elle s'entr'ouvre naturellement pour laisser place parmi ses descriptions abstraites comme par un trou percé dans la partie d'une chambre noire à la réalité extérieure ${ }^{110}$. Le " trou percé" a partie liée à " la trouée dans le décor ": le sens excède la "réalité » physique. La page " trouée " ne doit pas faire écran...

L'auteur affecte certes de se faire "concret ", notamment en étudiant la matérialité de la page. Le diplomate s'intéresse encore à l'architecture de la langue...

\section{L'architecture de la langue}

Selon Philippe Hamon, l'architecture en littérature devient forcément metalangage. Or, notre anarchiste chrétien érige volontiers le langage architectural en heuristique de la langue : « le français est [...] une leçon pratique d'architecture verbale. La première obligation pour un homme qui a la prétention de s'exprimer correctement dans notre langue, c'est d'apprendre à construire une phrase [...] à mesurer comme un ingénieur le ressort et la portée des différentes propositions incidentes qui viennent s'appuyer comme les courbes d'une voûte sur le fût solide de la proposition principale. Le français apprend à construire, car il est impossible d'apprendre à construire sa pensée [...] En exprimant les idées, nous les expérimentons ${ }^{111}$ [...] nous voyons ce qu'elles font, les conséquences qu'elles entraînent, les développements qu'elles exigent, les idées accessoires qu'elles suscitent ${ }^{112}$. L'architecture se transforme non seulement en outil euristique, mais aussi en panégyrique du français.

La calligraphie chinoise, magnifique expression de ce que doit être l'architecture, destinée à insuffler le mouvement à un objet inanimé, donnant ainsi du sens aux formes et des formes au sens, retentit sur le conférencier. François Cheng utilise pareillement dans son Dialogue l'architecture afin de définir «les vertus du français » : «si je devais décrire les vertus $d u$ français $[\mathrm{je}]$ dirais [...] qu'intrinsèquement il contient une série d'exigences $[\ldots]$ exigence d'une structure charpentée ${ }^{113}$. 
Le catholique errant confere même directement une troisième dimension aux mots, à la métaphore. "Le Poëte. - Pardon! pardon! pardon! ce n'est pas de ma faute! je ne savais pas! Où c'est-i114 que c'est que je suis-t-i? Danse-la-Nuit. - De l'autre côté, Monsieur, de la métaphore ${ }^{115}$." Fabuleuse, fantaisiste mise en espace de la parole...

Cependant, nous ne pouvons conclure sur Claudel architecte sans traiter de son urbanisme.

\section{Claudel urbaniste : «Ville la nuit ${ }^{116} »$}

Claudel, poète de la ville... L'âme piétonne permet d'appréhender la ville, d'aborder l'urbanisme et ses enjeux. Rappelons que la Connaissance loue le "perpétuel piéton ${ }^{117}$. De fait, le privilège de connaitre authentiquement la ville est dévolu au piéton. La sinophile Connaissance révèle la profession de foi en faveur du marcheur : pensons ${ }^{118}$ que l'ancienneté ${ }^{119}$ urbanistique chinoise $^{120} a$ influencé le poète. Pratique de piéton et éducation à la Chine valent propédeutique urbanistique : "Chang'an, la capitale des Tang, est dite "la plus grande ville $d u$ monde" [...] elle occupait une superficie d'environ 100 kilomètres carrés. Surpassant de loin le Pékin [...] des Qing, elle s'approchait du Pékin d'aujourd'hui. Les principaux boulevards de Chang'an sont larges de 100 à 150 mètres $»^{121}$.

Forum viarum ${ }^{122}$, la ville constitue un noud de contradictions, religieuses, sociales, le cas échéant explosif ${ }^{123}$, e.g. la Ville claudélienne. Aux Etats-Unis, le diplomate a déploré le "vécu du piéton », vérifié le déficit d'habitabilité, sinon des "villes tentaculaires", en tout cas des rues bostoniennes...

Plus dramatiquement, la Ville atteste la complexité affectant l'espace urbain; elle devient le lieu où les formes architecturales, politiques s'interpénètrent, expriment la ville, la saccagent, l'incendient, octroyant finalement à celle-ci un sens tragique. La cité est une entité : ce qu'elle est intrinsèquement transcende l'agrégation de ses éléments, dans la fusion, la destruction, l'immolation. Lambert manifeste en quelque manière ce que les sociologues appellent actuellement le caractère « sociofuge »124 de certaines conceptions urbanistiques : "Cherchant à se rejoindre, ils n'y peuvent parvenir;

Plus ils sont proches, plus le mouvement est précipité.

Tel est ce mouvement qu'il y a dans les villes ${ }^{125}$. » 
Nous songeons à Ping Hsin... « L'âme

Cette petite lueur

au cour du silence flamboie

au cour des villes s'efface $»^{126}$.

Quant à Claudel, il est sans ambages s'agissant de son pessimisme à l'encontre de la Ville: l'anticonformiste enseigne que celle-ci sent l'excrément et la tuerie... La conclusion de la cité ne peut qu'être l'apocalypse. Le personnage de Gérin dépeint l'apocalypse urbaine.

"Le soleil qui se couche, parmi l'effusion de plus de sang

Ne dévaste pas la Cité des Nues d'un trou et d'une ruine plus affreuse

Que la Ville humaine ne s'est abîmée dans la consommation des rêves.

Comme une feuille de papier où le feu avance et mord ${ }^{127}$,

C'est ainsi que, dix jours et dix nuits, dans la bise impitoyable, dans le feu qui flambe et qui ronfle,

Montagne de fumée dans le soleil, splendeur vive par les ténèbres,

Elle a brûlé tout entière ${ }^{128}$. »

Le dramaturge pressent le danger de trop nombreux slogans, (re)formulés par Anatole Kopp... " La vie nouvelle exigeait une ville nouvelle". Ou encore : "Changer la vie, changer la ville». Sur un autre plan, préfiguration d'Hiroshima ${ }^{129}$. "A travers les villes en flammes " ${ }^{130}$ constitue le bûcher des vanités...

Sans doute la vision claudélienne apocalyptique de la ville est-elle inspirée par l'exégèse à laquelle se livrait toujours davantage l'écrivain. Epître aux Hébreux : "car nous n'avons pas ici de ville permanente, nous cherchons la ville à venir ${ }^{131}$. »

Au total, la thématique architecturale, telle que nous l'avons analysée, est riche... Le détour - chinois — vaut volontiers retour à l'âme, à l'architecture intérieure... L'église : "Si un vase vu du dehors est beau, pourquoi n'y aurait-il pas aussi une beauté intérieure [...] une jouissance de la cavité1 ${ }^{132}$ ? " L'architecte, concepteur ou penseur contemporain, Jean Nouvel rejoint le poète : «l'essentiel de notre condition, de notre matérialité est de l'ordre de l'invisible. L'influence des théories quantiques, des théories fractales sur l'architecture ne peut pas être nulle puisque c'est la structure globale de notre espace qui est en cause. L'essentiel de nous-mêmes, l'essentiel de ces structures-là est de l'ordre de l'invisible ${ }^{133}$." 


\section{Claudel architecte}

\section{Notes}

1 Croire ou non au Bouddha est déterminé par le sens de l'éveil et la prédisposition. Si le Bouddha apparaissait, qu'on puisse le voir clairement avec les yeux charnels, alors, tout le monde irait apprendre la pratique du Bouddha, le changement de pensée ne serait plus en question. Il faut d'abord accepter, ensuite vous pourrez voir...

2 "La Cathédrale de Strasbourg ", Euvres en prose, Paris, Gallimard, 1989, p. 315 .

3 En fait, notre "Claudel architecte " constitue un terrain original : l'Intégrale claudélienne présentée du 25 juin au 31 décembre 2003 par le Théâtre du Nord-Ouest, à laquelle il nous fut offert de participer, n'a pas traité spécifiquement de l'architecture claudélienne, même dans le cadre de L'Eil écoute, dont les discours sur la peinture et la sculpture furent mis en valeur.

4 Paul Claudel, « Le Soulier de satin », Théâtre II, Paris, Gallimard, 1996, p. 690.

5 Théâtre II, Paris, Gallimard, 1989, p. 1534.

6 Traduction de Raymond Delambre.

7 Idem.

8 Le chrétien Claudel est forcément influencé par le Cantique des cantiques, qui fait de la bien-aimée un jardin clos. Ysé, trop ouverte et à l'origine du malheur, versus Prouhèze, jardin fermé à Rodrigue, malgré celui-ci, bien sûr, mais aussi et surtout, plus subtilement, nonobstant le désir féminin.

9 Chez le créateur de Prouhèze et d'Ysé, la position de l'homme est à genoux devant la femme, ce qui n'est sans doute pas pardonné par ses détracteurs...

10 Yang Xin, La beauté des pierres, Presses littéraires et artistiques de Shanghai, 2000, p. 41.

11 Quant aux éventuels sceptiques, notre réponse : tout comme votre estomac, vous ne sentez pas qu'il est en mouvement...

12 En général, on voit à travers les yeux charnels et ce sont justement ceux-ci qui obstruent le passage menant vers d'autres espaces; les yeux jouent un rôle d'écran; on peut voir seulement les choses existant dans l'espace matériel.

13 Paul Claudel, Paris, Gallimard, 1997, p. 224.

14 Lao Tseu, Tao Te King, Montréal, Bellarmin, 1989, p. 49.

15 Euvres en prose, p. 316.

16 Hauteur sous plafond. 


\section{Raymond Delambre}

17 Euvres en prose, p. 77-78.

18 Le succès idéologique de l'automobile et la ruse du marketing tendent à faire croire à celui qui se prétend un "pilote ", i.e. en fait ce que nous appelons un " chauffeur sans gages ", qu'il n'est pas sur des rails, alors que son cher véhicule n'est qu'un wagon, ou plutôt n'est pas même un wagon confortable - e.g. un wagon-salon. Pourvu que l'automobile ne soit pas un wagon-bar, un wagon-citerne...

19 Davantage tirés que poussés. Mieux vaut être tiré que poussé : tel est l'enseignement claudélien.

20 Ecrits, Paris, Hermann, 1991, p. 62.

21 Osons la formule «nous sommes tous des insulaires».

22 Sur moi-même, Arles, Editions Philippe Picquier, 2003, p. 73.

23 L'Oiseau noir dans le soleil levant, p. 235.

24 La Voie des Fleurs et des Oiseaux dans la tradition des Song, Paris, Phébus, 2000, p. 48.

25 Euvres en prose, p. 297.

26 Théâtre II, 1996, p. 1416.

27 On sait que Claudel se défie du freudisme, moderne secte, sinon religion.

28 Le feu couve sous le fonctionnaire, diplomate... Oserons-nous dire que le Christ claudélien est de quelque façon pasolinien?

29 Théâtre II, 1996, p. 1416.

30 Peintre qui fut aussi sculpteur et dessinateur. Il n'est pas indifférent qu'il étudia l'architecture.

31 Euvres en prose, p. 306.

32 Paul Claudel, Connaissance de l'Est, Paris, Gallimard, 1997, p. 91.

33 Euvres en prose, p. 233.

34 Heureusement, le critique d'art commémore cet artiste oublié...

35 Euvres en prose, p. 298.

36 Id., p. 305-306.

37 La perpendicularité a partie liée à l'art d'édifier - perpendiculum désigne le fil à plomb - et rejoint le concept de verticalité.

38 Nous avons déjà abordé le Claudel justicier : Raymond Delambre, «Le relais livre en campagne, outil de l'aménagement culturel du territoire ", Transversales, $\mathrm{n}^{\circ} 64,1998$, p. 6-11. 


\section{Claudel architecte}

39 Bâtiment et travaux publics.

40 Cuvres en prose, p. 304.

41 On sait que l'expression d'« arrangement » a fait florès.

42 Cuvres en prose, p. 304.

43 Id., p. 305.

44 Id., p. 304.

45 "Jean Charlot», id., p. 296.

46 La synesthésie, au sens de sunaisthèsis, est constamment (re)découverte par les experts et critiques ou experts critiques : e.g. les commentaires de l'exposition consacrée du 13 octobre 2004 au 17 janvier 2005 par les Galeries nationales du Grand Palais à Joseph Turner, James Whistler, Claude Monet.

47 Cuvres en prose, p. 297.

48 Théâtre II, 1989, p. 1529.

49 "Sur la musique », Euvres en prose, p. 160. Notre écrivain associe ainsi les differrents arts, le titre musical recouvrant arts plastiques, architecture.

50 Id., p. 311.

51 Ou plutôt enrichit ses définitions.

52 "Appendice », Euvres en prose, p. 137.

53 La vérité nous conduit à affubler d'une paire, a minima, de guillemets " réalité » et « concret».

54 Bien sûr, la référence à la pierre, à Pierre, est notamment d'obédience religieuse : la doctrine catholique convie à accepter Pierre, condition nécessaire - et non obstacle — à l'unité des chrétiens. L'Otage claudélien s'en fait l'écho. Certes, Bible elle-même non sans ambivalence, Jésus étant la «pierre d'achoppement». De toute façon, Georg Hegel nous enseigne que l'obstacle peut constituer non seulement un écueil, mais encore un appui...

55 Peintre, ami (1876-1945).

56 CEuvres en prose, p. 292.

57 «La Danse des morts », Théâtre II, 1996, p. 1266-1267.

58 En qualité de membre de différents jurys, nous sanctionnons l'usage abusif par les candidats de "car", conjonction de coordination : mais le poète a davantage de licence...

59 Théâtre II, 1996, p. 68.

60 Cuvres en prose, p. 74. 


\section{Raymond Delambre}

61 Le caractère chinois peut être considéré comme une entité.

62 «a Philosophie du livre », CEuvres en prose, p. 74.

63 Hélas, le catholique errant a raison d'alerter face aux gratte-ciel, même s'il n'est guère suivi : les fractales, redécouvertes récemment par lhomme occidental, sont même utilisées, voire détournées, "dé-tour-nées " afin d'ériger des tours toujours plus élevées... Ainsi, un gratte-ciel de cinq-cents mètres doit bénéficier d'un ciment à structure fractale.

64 Dont la fragilité fut dénoncée bien avant certain 11 septembre... Il est extraordinaire que les attaques - il ne s'agit pas de "simples" attentats ou, alors, il faudrait (re)qualifier l'acte de guerre à Pearl Harbour du 7 décembre 1941 d' « attentat», acte au demeurant «moins grave » que ceux commis le 11 septembre 2001 - n'aient guère remis en cause la construction des tours : symptôme de l'idéologie dominante et de sa prégnance, empêchant que ne soit questionné le consensus, notamment architectural. Pourtant, "la tour, prends garde de te laisser abattre "...

65 On sait que le département " révolutionnaire " constitua une arme de destruction massive à l'encontre des provinces d'Ancien Régime, les régions contemporaines quant à elles frelatant la notion de province.

66 «La Sagesse sur la parabole du festin », Théâtre II, 1996, p. 1206.

67 Jean-Marie Pérouse de Montclos, Histoire de l'architecture française, Paris, Editions Mengès, 1989, p. 184.

68 Architecture, Bruxelles, Pierre Mardaga, 1981, p. 534-535.

69 Première épître aux Corinthiens (X, 23).

70 «L'Argent et l'argenterie », Euvres en prose, p. 356.

71 Allusion aux Pierres de Venise...

72 Euvres en prose, p. 341. Le « girasol » tire son origine de l'italien « girasole», composé à partir de " girare »- tourner — et « sole», soleil : nous n’en dirons pas plus, respectant la sensibilité de Claudel à la magie des mots, à la différence des nombreux folliculaires dont l'obsession est d' "identifier ».

73 Toujours en rapport avec l'enseignement dispensé par Georg Hegel : de l'écueil à l'obstacle, de l'obstacle à l'appui...

74 Cuvres en prose, p. 344.

75 Porte religieuse.

76 Euvres en prose, p. 353.

77 « XII ${ }^{\mathrm{e}}$ et XIII ${ }^{\mathrm{e}}$ siècles », id., p. 335. 


\section{Claudel architecte}

78 Aspirant verrier...

79 Titre éponyme, Euvres en prose, p. 336.

80 L'eau est un motif chinois.

81 Autre oxymore : « eau solidifiée»...

82 Euvres en prose, p. 337.

83 Ne refusons pas le plaisir de citer une nouvelle fois : «c'est l'esprit qui contient le corps, et qui l'enveloppe tout entier. »

84 Cuvres en prose, p. 338. Nous constatons ici que notre grand voyageur résiste à la tendance esthétique - tentation - visant à redéfinir l'espace de l'épistémè, où il s'agirait d'assimiler les « belles-lettres » aux "beaux-arts » ou de leur substituer la notion d'art. Le poète demeure in fine conscient de son " infirmité » par rapport au plasticien...

85 Si l'on veut, le verre est le squelette, la lumière incarne la physiologie...

86 Nous sommes reconnaissant à Ming Delambre-Zhou, architecte internationale, avec laquelle nous avons parcouru les champs sémantiques - et affectifs - voire (électro)magnétiques de l'illumination, de l'éblouissement : nos visites effectuées à Notre-Dame de l'Arche d'Alliance (à Paris, dans le quinzième arrondissement) nous ont permis d'apprécier la croix de cette église, croix de lumière sans support physique apparent, sans épaisseur.

87 "Appendice », Euvres en prose, p. 137.

88 Paul Claudel, L'Annonce faite à Marie (première version), Théâtre II, 1996, p. 67.

89 L'Oiseau noir, p. 234.

90 Umberto Eco, La structure absente, Paris, Mercure de France, 1988, p. 277-278.

91 « José-Maria Sert et sa cathédrale », Euvres en prose, p. 288-289.

92 Ecrire avec un style si chic explique probablement les nombreux détracteurs, forcément jaloux, de Claudel.

93 Nous pensons à Henri Alekan, parsemant d'étoiles certaines marches de Paris et mariant cinéma et «éclairage urbain »...

94 «Appendice», Euvres en prose, p. 136.

95 Plutôt que de simples silences métaphoriques, entendons ici un silence décliné selon la synesthésie.

96 Euvres en prose, p. 134.

97 « L'église maison de prière », id., p. 135. 


\section{Raymond Delambre}

98 Admirons le poète même lorsqu'il s'adonne à la prose et son audace, enjolivant l'insinuation du vulgum pecus, sensible au gossip...

99 « Appendice », Euvres en prose, p. 135.

100 P. 30.

101 "XII et XIII siècles ", Euvres en prose, p. 328.

102 Id., p. 73.

103 P. 155.

104 «Vent» et «eau» (traduction littérale).

105 Euvres en prose, p. 75.

106 «La Philosophie du livre », id., p. 76.

107 Id., p. 76-77.

108 Seul un point final devrait suivre, et certes pas une virgule, non plus qu'un " et »...

109 Le regret justifie l'antiphrase.

110 Euvres en prose, p. 81.

111 L'expérimentation des idées selon Claudel est en rapport avec le « drame d'étude ", syntagme qualifiant l'écriture dramatique.

112 Sur la langue française. Discours culturel non sans rapport avec l'appétence claudélienne pour le christianisme : la culture française est gréco-latine surtout latine - et christianisée, inséparable de celui-ci.

113 Presses artistiques et littéraires de Shanghai, 2002, p. 34-35.

114 Claudel, devenu parisien, demeurant amateur de la province, force volontiers le trait paysan.

115 Théâtre II, 1996, p. 1325.

116 Connaissance, p. 33.

117 P. 51.

118 Devant les Vitraux, le catholique errant lui-même offre quelque liberté à son lecteur. "Il y a en gestation un vocabulaire qui refuse en se précisant d'exclure aucune interprétation » ("XII ${ }^{\mathrm{e}}$ et XIII ${ }^{\mathrm{e}}$ siècles ", Euvres en prose, p. 328).

119 E. g 2004 a marqué le millénaire de la fondation pour Jingdezhen. Cette ville, loin d'être la plus ancienne cité chinoise, capitale mondiale de la porcelaine et dont la prodigieuse croissance est associée au développement de cet art, étonna l'Europe du XVIII ${ }^{\mathrm{e}}$ siècle par le degré de spécialisation atteint dans la division du travail. 


\section{Claudel architecte}

120 L'Asie donne effectivement des leçons d'urbanisme (et d'urbanité). Les conventions statistiques varient fortement : alors que le seuil français de la ville n'est que de 2000 habitants, la ville japonaise - shi- ne commence en principe qu'à partir de 50000 âmes.

121 Tang Ke Yang, Chanson de nuit, 2000, Presses littéraires et artistiques de Shanghai, p. 23.

122 Fourvière.

123 La ville comme condensateur social...

124 Censé s'attacher à l'urbanisme en damier, mosaïque : non sans rapport avec l'expérience claudélienne de la ville américaine. Ceci étant, à la décharge des Etats-Unis, le plan en damier — plan hippodaméen — n'a pas été inventé par un moderne Américain, mais établi par l'architecte Hippodamus de Millet. Certes, on pourrait démontrer que les Etats-Unis constituent les héritiers du classicisme, voire son conservatoire... Au demeurant, le plan orthogonal ou quadrillé ne se retrouve pas seulement dans les pays "neufs ": ainsi à Barcelone, selon le projet d'Ildefonso Cerdà (1860).

125 Paul Claudel, La Ville (deuxième version), Paris, Mercure de France 1982, p. 10.

126 François Cheng, Entre source et nuage, Paris, Albin Michel, 2002, p. 174.

127 Nous relevons la référence au livre pour décrire la ville, référence métaphorique notamment, ainsi que la référence au Livre : l'Apocalypse de Jean se réfère aussi à l'écrit afin de décrire l'univers...

$128 \mathrm{La}$ Ville (deuxième version), p. 77.

129 Sans oublier Nagasaki.

130 L'Oiseau noir, p. 165.

131 La Bible, Paris, Gallimard, 1994, p. 781.

132 «Appendice », Euvres en prose, p. 138.

133 Conférence donnée au XIX ${ }^{\mathrm{e}}$ Congrès de l'Union internationale des Architectes à Barcelone, le 6 juillet 1996. Si le bâtiment est important aux yeux de Claudel, le texte importe au nouvel architecte du Havre : rappelons que Jean Nouvel présenta, pour l'obtention de son diplôme d'architecte reconnu par le gouvernement, un texte, et non une esquisse, non plus qu'une maquette. 